\title{
Análise descritiva do desempenho em uma prova de 100 m nado livre feminino baseada em variáveis biomecânicas
}

CDD. 20.ed. 796.023

797.2

\author{
Breno Quintella FARAH* \\ Windson Carlos Motta SILVA* \\ Marcos André SANTOS* \\ Wilson Viana de Castro MELO*
}

${ }^{*}$ Escola Superior de

Educação Fisica, Uni-

versidade de Pernam-

buco.

\section{Resumo}

Na natação, atualmente, a avaliação do desempenho dos nadadores, apóiam-se em diversas metodologias de observação da competição. As variáveis biomecânicas representam um importante recurso para análise da performance esportiva por serem indicadores da eficiência do nado, com aplicação simples e objetiva. 0 objetivo deste estudo foi realizar uma análise descritiva do desempenho na prova de 100 metros nado Livre feminino, baseada nas variáveis: comprimento de braçada (CB), frequência de braçada (FB), velocidade de nado (VN), índice de nado (IN), velocidade média escalar (Vm), tempo de saída (Ts) e tempo de virada (TV), a fim de observar o comportamento e influências na performance. Foram capturadas imagens por uma câmera mini-DV, analisadas por um "software" editor de vídeo. As variáveis $C B$, VN e IN foram encontradas indiretamente, sem considerar as fases de saída e virada. Ts e Tv foram obtidos através da linha do tempo do software. A análise de dados mostra para atleta 1: CB 2,00 m, FB 0,89 Hz, VN 1,78 m/s e IN 3,56 m²/s; atleta 2: CB 1,82 m, FB 0,97 $\mathrm{Hz}, \mathrm{VN} \mathrm{1,76} \mathrm{m/s,} \mathrm{IN} \mathrm{3,20} \mathrm{m²/s;} \mathrm{atleta} \mathrm{3:} \mathrm{CB} \mathrm{1,91} \mathrm{m,} \mathrm{FB} \mathrm{0,92} \mathrm{Hz,} \mathrm{VN} \mathrm{1,75} \mathrm{m/s,} \mathrm{IN} \mathrm{3,32} \mathrm{m²/s.} \mathrm{Conclui-se} \mathrm{que:} 0$ desempenho final apresentou comportamentos similares nos três primeiros trechos da prova indicando uma influência semelhante dessas variáveis sobre o desempenho das atletas. No último quarto da prova as variáveis apresentam maior interferência na performance final, sendo este decorrente de uma possível diminuição da eficiência mecânica do nado devido ao aumento do arrasto e redução da força propulsiva.

Unitermos: Biomecânica; Natação; Comprimento de braçada; Frequência de braçada; Índice de nado.

\section{Introdução}

Na natação, atualmente, a avaliação do desempenho dos nadadores, apóiam-se em diversas metodologias de observação da competição (TRINITY, PAHNKE, REESE \& COYLE, 2006), em que cada uma orienta-se a partir de um protocolo específico, a fim de diagnosticar parâmetros cinemáticos julgados convenientes na determinação do comportamento e desempenho do nadador (Williams \& Kendall, 2007).

Dentro desta perspectiva observa-se que tanto pesquisadores quanto atletas e técnicos demonstram um grande interesse pelos fatores determinantes do desempenho na natação, por estes serem ferramentas que podem ajudar a evoluir as estratégias de treinamento em busca dos melhores resultados (CASTRO, 2007).

Um bom desempenho na natação está diretamente relacionado com os fatores biomecânicos que envolvem alinhamento dinâmico do corpo no meio líquido (Colwin, 2000); nível de performance; habilidade técnica e características antropométricas dos atletas (Caputo, Oliveira, Denadai \& Greco, 2006; Franken, Carpes \& Castro, 2007), além da superação da fadiga provocada pelos esforços anaeróbios láticos (MAGLischo, 1999), como também gênero e idade (CAPUTO et al., 2006)

São pontos de interesse inerentes ao estudo da biomecânica da natação as manifestações técnicas do nado, a descrição e a quantificação das forças de resistência e propulsão (Craig Junior, SkeHan, PawelczyK \& Boomer, 1985; Franken, Carpes \& Castro, 2007; Liu, Hay \& Andrews, 1993). Toussaint e Hollander (1994) afirmam que a identificação e a mensuração de fatores biomecânicos 
são importantes para o desempenho do nado, por terem grande influência sobre o arrasto e/ou desenvolvimento da força propulsiva (Craig Junior et al., 1985).

Dentre as variáveis biomecânicas para a análise do desempenho dos atletas, a frequência de braçada (FB), o comprmento de braçada (CB), a velocidade média de nado $(\mathrm{VN})$ e o índice de nado (IN), são as mais utilizadas pelos treinadores, devido à simplicidade e objetividade das mesmas (CASTRO, GUImarães, Moré, Lammerhirt \& Marques, 2005).

Para Silva, Silva, Reis, Reis, Marinho, Carneiro e AIDAR (2007) a análise da competição consiste em medir os diferentes componentes da prova em seu tempo total, ou seja, tempo de saída (Ts), tempo de nado, tempo de virada (Tv) e tempo de chegada. Outro fator de desempenho, diz respeito à velocidade média escalar $(\mathrm{Vm})$, que é representada pela relação entre o espaço percorrido e o tempo total gasto para realização do percurso.

Deste modo, este estudo tem por objetivo realizar uma análise descritiva do desempenho das três atletas mais bem colocadas na prova final dos 100 m nado livre feminino de uma competição internacional de alto nível, a qual envolveu várias atletas do continente americano, a fim de observar o comportamento das variáveis $\mathrm{FB}, \mathrm{CB}, \mathrm{VN}, \mathrm{IN}, \mathrm{Vm}$, Ts e Tv, durante a prova e a suas influências na performance.

\section{Metodologia}

Este estudo foi aprovado pelo Comitê de Ética em Pesquisa com Seres Humanos da Universidade de Pernambuco (CEPE/UPE) em consonância com as resoluções do Conselho Nacional de Saúde (CNS). A prova final dos 100 metros nado Livre feminino dos Jogos Pan-americanos, Rio 2007, realizada em uma piscina olímpica medindo $50 \mathrm{~m}$ x $25 \mathrm{~m} \times 3 \mathrm{~m}$ com 10 raias, foi filmada por meio de uma câmera (mini-DV, marca JVC-9800) com frequência de $60 \mathrm{~Hz}$ e analisada pelo "software" editor de vídeo Sony Vegas 7.0.
A câmera foi apoiada em um suporte de fixação vertical (tripé) e posicionada na parte superior das arquibancadas destinadas ao público, de forma a possibilitar a filmagem de todas as atletas, simultaneamente, por meio da técnica "panning" em toda extensão da piscina, em sua vista superior, garantindo, assim, a não superposição das imagens das atletas, sendo utilizadas como referência as marcaçóes das raias divisórias, a partir das medidas $15 \mathrm{~m}, 25 \mathrm{~m}$ e $50 \mathrm{~m}$ (FIGURA 1). Foram analisados apenas os desempenhos das três atletas que apresentaram melhor colocação na prova.

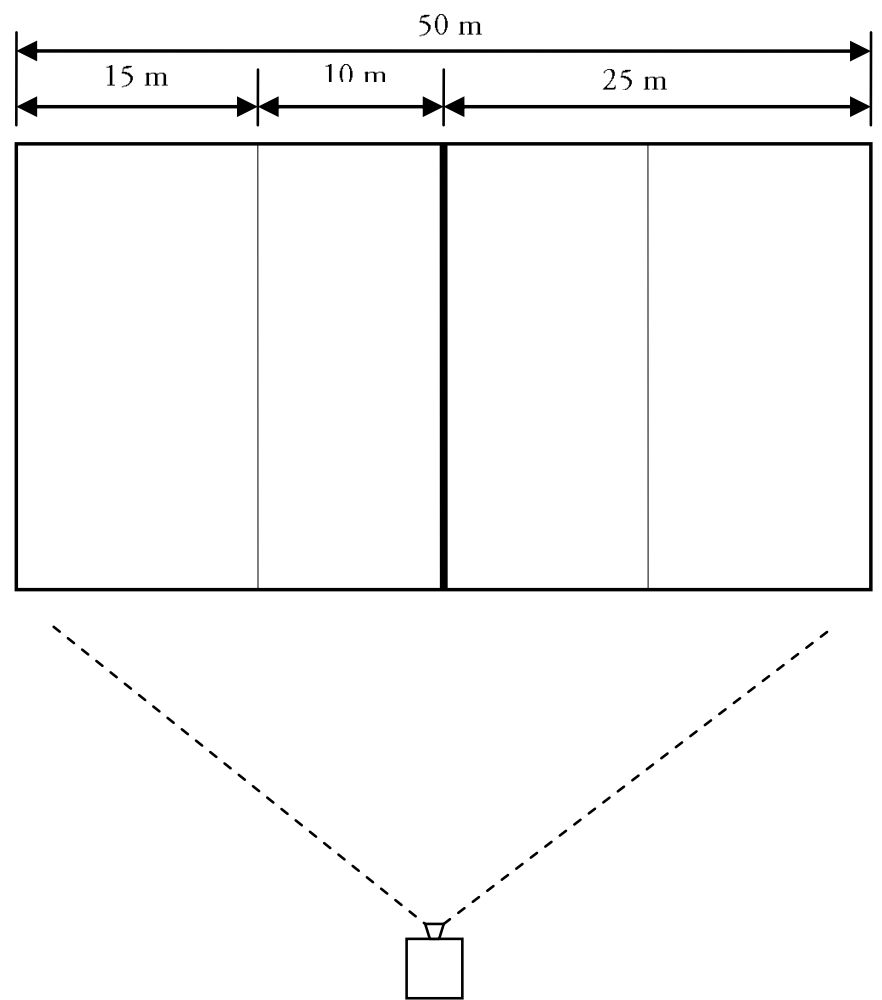

FIGURA 1 - Posicionamento da câmera em relação à piscina. 
A linha de tempo do "software" foi iniciada e finalizada de acordo com aos tempos oficiais fornecidos pela organização da competição.

A FB é o número médio de ciclos de braçada (Chollet, Chalies \& Chatard, 2000), expressa em ciclo por segundo ou Hertz. Para a contagem de um ciclo de braçada, utilizou-se como referência o momento de entrada de uma das mãos na água e o retorno da mesma mão (CASTRO et al., 2005). Ela foi realizada de forma contínua e dividida de acordo com as marcações estabelecidas, sendo considerado como menor unidade de medida meio ciclo de braçada.

As contagens foram feitas por três observadores devidamente treinados, quando houve divergências, o processo foi repetido até o estabelecimento de um número comum entre estes.

$\mathrm{O}$ CB é a distância horizontal média percorrida durante a execução de um ciclo completo de braçada, em metros (MAGLISCHO, 1999).

As variáveis $\mathrm{FB}$ e $\mathrm{CB}$ foram obtidas em momentos cíclicos do nado, em dois trechos distintos, a fim de eliminar os efeitos da saída e virada e não superestimar os valores relativos ao CB (SMith, NorRIs \& HogG, 2002). Desta forma, considerou-se dos 15 aos 35 metros e dos 65 aos 85 metros. Para cada trecho, o valor da FB foi determinado pela divisão do número de ciclos de braçadas pelo tempo de realização, fornecido pelo "software". O CB foi determinado indiretamente para

\section{Resultados}

Os valores dos ciclos de braçadas efetuados por cada atleta nos trechos de 15 a 35 e 65 a 85 metros com seus respectivos tempos, o total de ciclos no decorrer da prova e velocidades médias são apresentados na TABELA 1. De acordo com o resultado final, sendo a atleta 1 a primeira colocada, a atleta 2 a segunda colocada e a atleta 3 a terceira colocada.

TABELA 2 mostra o comportamento das variáveis: tempo de saída, tempo de virada, comprimento de braçada, frequência de braçada, velocidade de nado e cada trecho, por meio do produto do período (inverso da frequência) "versus" a velocidade média de cada percurso. Os valores finais da FB e CB foram obtidos através da média aritmética dos valores determinados em cada trecho.

$\mathrm{O}$ produto entre as variáveis, $\mathrm{FB}$ e $\mathrm{CB}$, fornece a $\mathrm{VN}$, sendo expressa em metro por segundo, desconsiderando os efeitos propulsivos da saída e virada (Caputo, Lucas, Greco \& Denadai, 2000; Hay \& GuimarÃES, 1983), enquanto que o IN decorre do produto entre CB e VN (CAPUTO et al., 2000), em metro quadrado por segundo.

A Vm da prova expressa em metros por segundo foi obtida a partir da relação entre a distância e o tempo oficial da prova. Por interesse desse estudo, optou-se por determinar, também, a $\mathrm{Vm}$ por trechos: 0-25 m; 25-50 m; 50-75 m e 75-100 m sendo utilizadas para esse fim, as demarcações pré-estabelecidas, em que a relação espaço/tempo foi obtida pela distância de cada trecho e o tempo necessário para percorrê-la, esse tempo foi obtido por meio do "software".

O Ts foi estabelecido no momento em que a atleta perde o contato com o bloco até o instante em que ela inicia o primeiro ciclo de braçada e o $\mathrm{Tv}$ a partir do momento em que ela termina a última braçada e realiza a virada propriamente dita até o reinicio do novo ciclo de braçada. Ambos também foram determinados por meio do "software".

índice de nado, correspondendo à média dos dois trechos de 20 metros ( 15 a 35 metros e 65 a 85 metros).

A TABELA 3 mostra os valores das variáveis ciclos de braçada, velocidade média e tempo em trechos da prova de 0-25 m, 25-50 m, 50-75 m e 75-100 m, respectivamente

A FIGURA 2 representa graficamente a variação da velocidade de cada atleta durante o decorrer da prova, relativamente a $25,35,50,75,85$ e 100 metros.

TABELA 1 - Ciclos de braçadas em trechos de 20 metros com seus respectivos tempos, o total de ciclos no decorrer da prova e velocidades médias.

\begin{tabular}{ccccccc}
\hline Atletas & $\begin{array}{c}\text { Ciclos de braçadas } \\
\mathbf{1 5} \mathbf{~ m ~ - ~ 3 5 ~} \mathbf{~ m}\end{array}$ & Tempo $(\mathbf{s})$ & $\begin{array}{c}\text { Ciclos de braçadas } \\
\mathbf{6 5} \mathbf{~ m ~ - ~ 8 5 ~} \mathbf{~ m}\end{array}$ & Tempo $(\mathbf{s})$ & $\begin{array}{c}\text { Ciclos de braçadas } \\
\text { totais }\end{array}$ & $\begin{array}{c}\text { Velocidade } \\
\text { média }(\mathbf{m} / \mathbf{s})\end{array}$ \\
\hline 1 & 10 & 10,94 & 10 & 11,47 & 42 & 1,78 \\
2 & 10,5 & 10,95 & 11,5 & 11,71 & 46,5 & 1,77 \\
3 & 10,5 & 11,08 & 10,5 & 11,75 & 42 & 1,75 \\
\hline
\end{tabular}


FARAH, B.Q. et al.

TABELA 2 - Variáveis: tempo de saída, tempo de virada, comprimento médio de braçada, frequência média de braçada, velocidade de nado e índice de nado.

\begin{tabular}{ccccccc}
\hline Atletas & Ts $(\mathbf{s})$ & Tv $(\mathbf{s})$ & CB $(\mathbf{m})$ & FB $(\mathbf{H z})$ & VN $(\mathbf{m} / \mathbf{s})$ & $\mathbf{I N}\left(\mathbf{m}^{2} / \mathbf{s}\right)$ \\
\hline 1 & 3,81 & 3,14 & 2,00 & 0,89 & 1,78 & 3,56 \\
2 & 3,54 & 3,10 & 1,82 & 0,97 & 1,76 & 3,20 \\
3 & 5,04 & 3,40 & 1,91 & 0,92 & 1,75 & 3,32 \\
\hline
\end{tabular}

TABELA 3 - Ciclos de braçada, velocidade média e o tempo por trechos da prova.

\begin{tabular}{clccc}
\hline Trechos & \multicolumn{1}{c}{ Variáveis } & Atleta $\mathbf{1}$ & Atleta 2 & Atleta 3 \\
\hline \multirow{2}{*}{$0-25$} & Ciclos & 07 & 09 & 07 \\
& Vm $(\mathrm{m} / \mathrm{s})$ & 2,08 & 2,05 & 2,09 \\
& Tempo $(\mathrm{s})$ & 12,01 & 12,21 & 11,98 \\
\multirow{2}{*}{$25-50$} & Ciclos & 12 & 12,5 & 12 \\
& Vm $(\mathrm{m} / \mathrm{s})$ & 1,69 & 1,7 & 1,68 \\
& Tempo $(\mathrm{s})$ & 26,77 & 26,93 & 26,87 \\
$50-75$ & Ciclos & 10 & 11 & 09 \\
& Vm $(\mathrm{m} / \mathrm{s})$ & 1,84 & 1,79 & 1,79 \\
& Tempo $(\mathrm{s})$ & 40,31 & 40,51 & 40,71 \\
$75-100$ & Ciclos & 13 & 14 & 14 \\
& Vm $(\mathrm{m} / \mathrm{s})$ & 1,68 & 1,63 & 1,65 \\
& Tempo $(\mathrm{s})$ & 55,17 & 55,78 & 55,84 \\
\hline
\end{tabular}

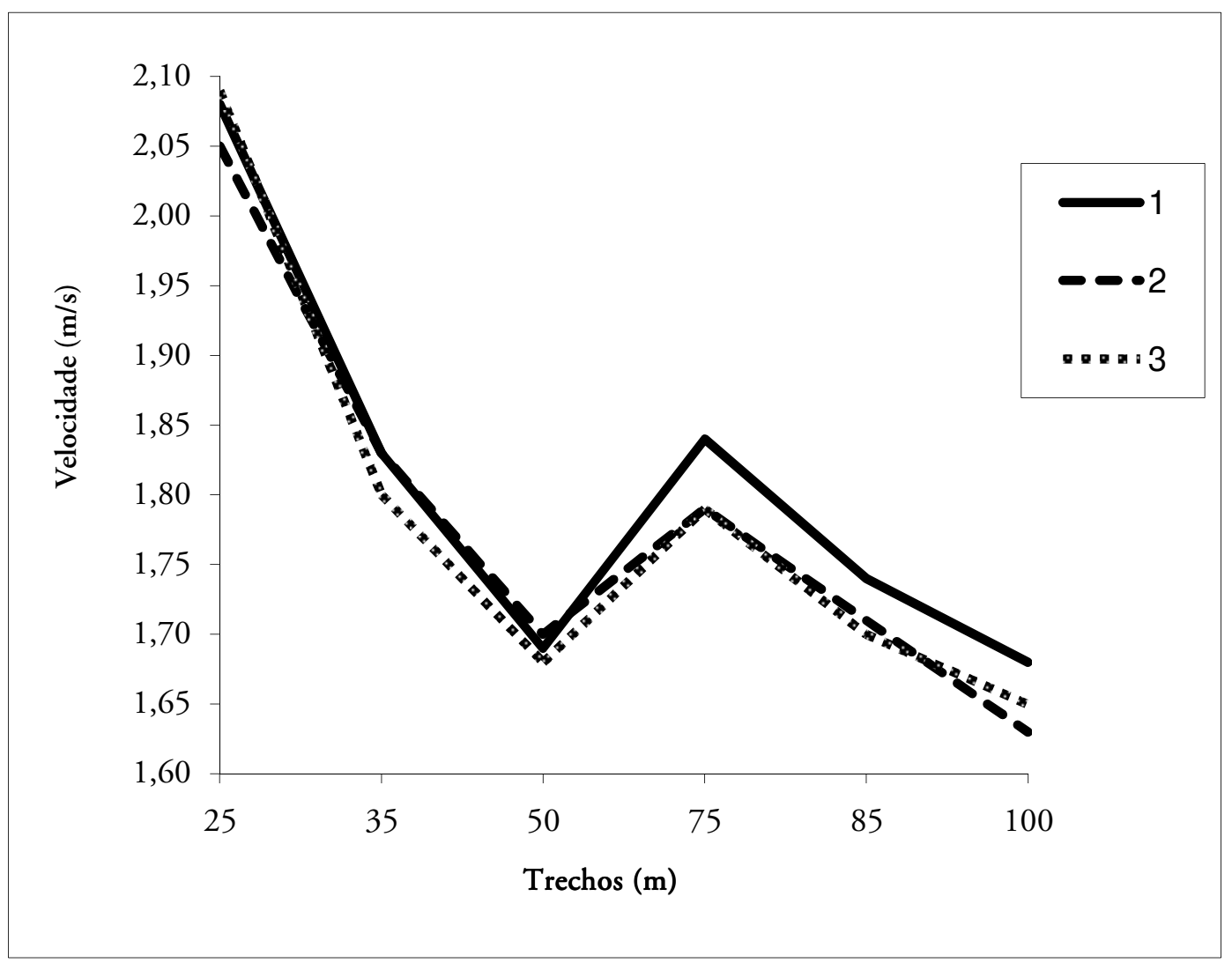

FIGURA 2 - Variação da velocidade média das atletas por trecho da prova.

466 • Rev. bras. Educ. Fís. Esporte, São Paulo, v.24, n.4, p.463-69, out./dez. 2010 


\section{Discussão}

Esse estudo procurou analisar de forma descritiva o desempenho das três primeiras colocadas numa prova de 100 m nado Livre, que contou com a participação de atletas de alto nível do continente americano, baseandose nas variáveis $\mathrm{FB}, \mathrm{CB}, \mathrm{VN}, \mathrm{IN}, \mathrm{Vm}$, Ts, Tv.

Os dados apresentados na TABELA 1 expressam o desempenho geral das atletas durante a prova. É possível observar que a atleta 1 e a atleta 3 realizaram a mesma quantidade de ciclos de braçada totais, porém a velocidade média final, entre elas, foi diferente. Em relação à atleta 2, observa-se que esta apresentou a maior quantidade de ciclos de braçada, porém sua velocidade média na prova coincidiu com a atleta 3 e foi inferior a atleta 1 .

Essa diferença entre as velocidades pode ser explicada pelo fato das atletas 2 e 3 apresentarem menores valores no que se refere ao $\mathrm{CB}$, quando comparadas à atleta 1 (TABELA 2), visto que essa variável representa um importante indicativo de desempenho técnico (CASTRO et al., 2005) e possibilita melhor eficiência propulsiva do nado (CAPUTO et al., 2000, 2006).

As variáveis biomecânicas responsáveis pela performance do nado são mostradas na TABELA 2. Em relação ao FB, CB, VN, IN, a atleta 1 apresentou melhores valores, o que pode ser observado através $\mathrm{da}$ variável $\mathrm{VN}$, a qual reflete a relação entre $\mathrm{CB}$ e $\mathrm{FB}$ e não sofre interferência da saída, virada e chegada. A literatura afirma que um decréscimo ou a manutenção da FB e um aumento no CB representam uma maior eficiência propulsiva ou um decréscimo no arrasto, acarretando, assim, um aumento na $\mathrm{VN}$ e um menor gasto na energia requerida para o nado (CAPUTO et al., 2000; CAstro et al., 2005; Gatti, Erichsen \& Melo, 2004; Maglischo, 1999; YanaI, 2003). Esses índices são os mais utilizados em estudos relacionados com a análise da performance e da eficiência técnica dos nadadores e podem ser influenciados pelos estados de fadiga (GATti, ERIChSEN \& Melo, 2004).

A atleta 2 foi a que apresentou menor Tv, quando comparada com as demais. Esta redução no tempo pode estar relacionada com a velocidade de aproximação da quadra de virada, juntamente com a coordenação entre os movimentos do nado e a técnica da virada (Platonov, 2004).

Constata-se que a atleta 3, quando comparada com as demais, apresentou o maior Ts. Mesmo com esta diferença entre os valores, observa-se que o seu desempenho pode ser explicado pelo melhor aproveitamento na fase submersa, acarretando menor tempo no primeiro quarto da prova (TABELA 3). Essa fase é de extrema importância para obtenção dos melhores resultados nas provas de 100 m (Platonov, 2004), já que ela representa aproximadamente 5\% do tempo total da prova, e um aprimoramento técnico representa decréscimos de até 0,2 segundos (MAGLISCHO, 1999).

Juergens, Rose, SMith e Calder (1999) ratifica a importância da variável Ts para obtenção de um melhor desempenho nas provas de $50 \mathrm{~m}$ nado Livre. Esses autores relatam existir uma diferença compreendida entre 0,1 a 0,16 segundos quando analisada a performance do segundo e do sétimo colocado nesta prova, o que sugere a importância da eficiência mecânica e velocidade de saída para o nadador.

$\mathrm{Na}$ TABELA 3, foi evidenciado que nos primeiros 25 metros da prova o melhor desempenho pode ser atribuído à atleta 3. Apesar de ter apresentado o mesmo número de ciclos de braçada em relação à atleta 1, essa obteve a maior Vm durante o percurso, o que pode ser atribuído ao melhor aproveitamento na fase de saída, observado na TABELA 2. O trecho da prova compreendido entre 25-50 metros demonstra, que as atletas apresentam um declínio natural $\mathrm{da} \mathrm{Vm}$, relativo à diminuição do deslize proporcionado pela saída. A atleta 2 neste trecho apresenta um discreto aumento na $\mathrm{Vm}$, o que possibilitou a diminuição da diferença expressa no primeiro quarto da prova.

No terceiro quarto da prova, que compreende o trecho entre 50-75 metros, a atleta 2 supera a atleta 3, este fato pode ser atribuído à diferença observada no Tv dessas atletas. Embora as atletas 2 e 3 tenham apresentado o mesmo valor na $\mathrm{Vm}$, a atleta 2 realizou maior número de ciclos de braçada, o que acarreta o decréscimo nos valores relativos ao $\mathrm{CB}$, aumentando assim, a energia requerida para o nado (MAGLISCHO, 1999).

É notável que no último trecho da prova, as atletas apresentam um maior número de ciclos de braçada, refletindo uma diminuição no desempenho do nado. Porém dentre as atletas, a atleta 3 foi a que apresentou a menor perda de desempenho, embora tenha tido um maior $T v$, já a atleta 2 foi a que apresentou o maior declínio.

Deste modo, como representado na FIGURA 2, as atletas 1 e 3 apresentam o mesmo perfil de nado durante os três últimos quartos da prova, expresso pelo comportamento das curvas descritas, observando-se apenas uma defasagem temporal nos dois últimos trechos da prova.

Considerando as dificuldades para análise das variáveis biomecânicas na Natação e as limitações metodológicas inerentes a este estudo, foi possível 
concluir, com base nos resultados encontrados que: o desempenho observado entre as atletas apresentou comportamentos similares nos três primeiros trechos da prova o que parece existir uma influência semelhante dessas variáveis sobre o desempenho final das atletas. Porém no último quarto da prova as variáveis analisadas passam a apresentar uma maior interferência na performance final, o que pode estar associado à relação entre as variáveis analisadas e a possível diminuição da eficiência mecânica do nado devido ao aumento do arrasto e redução da força propulsiva.

As informaçôes obtidas através deste estudo permitem analisar o desempenho técnico de atletas, realizar comparaçõos de resultados anteriores (individual e/ou em grupos) e também acompanhar a evolução esportiva.

\section{Abstract}

Descriptive analysis of performance in the female 100 meters free swimming based on biomechanics variables

The performance evaluation of swimmers is based on many methodologies of watching the competition. Biomechanical variables represent an important resource for analyzing the performance of swimmers because they are simple and objective indicators of swimming efficiency. The objective of this research was to observe how biomechanical variables affect swimmers' performance in of the female 100 meters freestyle in order to observe the behavior and influence stroke length (SL), stroke frequency (SF), swimming velocity (SV), swimming index (SI), average speed (AS), time of departure (Td), time of turning (Tt). On swimming performance images were captured using a mini-DV camera, which were subsequently analyzed with the help of a software video editor. The variables SL, SV and SI were indirectly measured, without considering start and turning phases. Td and Tt were obtained through the timeline of the software. Data analysis shows for athlete $1: \mathrm{SL}=2.00 \mathrm{~m}, \mathrm{SF}=0.89 \mathrm{hz}, \mathrm{SV}=1.78 \mathrm{~m} / \mathrm{s}, \mathrm{SI}=3.56 \mathrm{~m}^{2} / \mathrm{s}$, athlete 2: $\mathrm{SL}=1.82 \mathrm{~m}, \mathrm{SF}=0,97 \mathrm{~Hz}, \mathrm{SV}=1.76 \mathrm{~m} / \mathrm{s}, \mathrm{SI}=3.20 \mathrm{~m}^{2} / \mathrm{s}$, athlete $3: \mathrm{SL}=1.91 \mathrm{~m}, \mathrm{SF}=0.92 \mathrm{~Hz}, \mathrm{SV}=1.75$ $\mathrm{m} / \mathrm{s}, \mathrm{SI}=3.32 \mathrm{~m}^{2} / \mathrm{s}$. We concluded that the final performance presented similar behavior in the first three parts of the race, indicating a similar influence of these variables on the performance of athletes. In the last quarter of the race showed that variables have greater interference in the final performance, this probably due to a possible decrease in mechanical efficiency of swimming due to increase in drag and reduction in propulsive force.

UnITERMS: Biomechanics; Swimming; Stroke length; Stroke frequency; Swimming index.

\section{Referências}

CAPUTO, F.; LUCAS, R.D.; GRECO, C.C.; DENADAI, B.S. Características da braçada em diferentes distâncias no estilo crawl e correlações com a performance. Revista Brasileira Ciência e Movimento, São Caetano do Sul, v.8, n.3, p.7-13, 2000. CAPUTO, F.; OLIVEIRA, M.F.M.; DENADAI, B.S.; GRECO, C.C. Fatores intrínsecos do custo energéticoda locomoção durante a natação. Revista Brasileira de Medicina e Esporte, São Paulo, v.12, n.6, p.399-404, 2006.

CASTRO, F.A.S. Determinantes do desempenho para prova de 200 m nado livre. 2007. 152 f. Tese (Doutorado) Universidade Federal do Rio Grande do Sul, Porto Alegre, 2007.

CASTRO, F.A.S.; GUIMARÃES, A.C.S.; MORÉ, F.C.; LAMMERHIRT, H.M.; MARQUES, A.C. Cinemática do nado "crawl” sob diferentes intensidades e condiçōes de respiração de nadadores e triatletas. Revista Brasileira de Educação Física e Esporte, São Paulo, v.19, n.3, p.223-32, 2005.

CHOLLET, D.; CHALIES, S.; CHATARD, J.C. A new index of coordination for the crawl: description and usefulness. International Jornal of Sports Medicine, Stuttgard, v.21, n.1, p.54-9, 2000.

COLWIN, C.M. Nadando para o século XXI. São Paulo: Manole, 2000.

CRAIG JUNIOR, A.B.; SKEHAN, P.L.; PAWELCZYK, J.A.; BOOMER, W.L. Velocity, stroke rate and distanve per stroke during elite swimming competition. Medicine and Science in Sports and Exercise, Madison, v.17, n.6, p.625-34, 1985. 
FRANKEN, M.; CARPES, F.P.; CASTRO, F.A.S. Cinemática do nado crawl, características antropométricas e flexibilidade de nadadores universitários. In: CONGRESSO BRASILEIRO DE CIÊNCIAS DO ESPORTE, 15.; CONGRESSO INTERNACIONAL DE CIÊNCIAS DO ESPORTE, 2., 2007, Recife. Anais... Recife: CBCE, 2007.

GATTI, R.G.O.; ERICHSEN, O.A.; MELO, S.I.L. Respostas fisiológicas e biomecânicas de nadadores em diferentes intensidade de nado. Revista Brasileira de Cineantropometria \& Desempenho Humano, Florianópolis, v.6, n.1, p.26-35, 2004. HAY, J.G.; GUIMARÃES, A.C.S. A quantitative look at swimning biomechanics. Swimnig Technique, North Hollywood, v.20, p.11-7, 1983.

JUERGENS, C.A.; ROSE, D.J.; SMITH, G.A.; CALDER, C.A. A kinetic and kinematic comparison of the grab and track starts in competitive swimming. Medicine and Science in Sports and Exercise, Madison, v. 31, n.5, p.0-0, 1999.

LIU, Q.; HAY, J.G.; ANDREWS, J.G. Body roll and handpath in freestyle swimming: an experimental study. Journal of Applied Biomechanics, Champaign, v.9, p.238-53, 1993.

MAGLISCHO, E.W. Nadando ainda mais rápido. São Paulo: Manole, 1999.

PLATONOV, V.N. Treinamento desportivo para nadadores de alto nível. São Paulo: Phorte, 2004.

SILVA, A.; SILVA, F.; REIS, A.; REIS, V.; MARINHO, D.; CARNEIRO, A.; AIDAR, F. Análise das componentes da prova como ponto de partida para a definição de objectivos na natação na categoria de cadetes. Revista Portuguesa de Ciência do Desporto, Porto, v.7, n.2, p.189-201, 2007.

SMITH, D.J.; NORRIS, S.R.; HOGG, J.M. Performance evaluation of swimmers: scientific tools. Sports Medicine, Auckland, v.32, n.9, p.539-54, 2002.

TOUSSAINT, H.M.; HOLLANDER, A.P. Energetics of competitive swimming. Implications for training programmes. Sports Medicine, Auckland,, v. 18, n. 6, p. 384-405, Dec 1994.

TRINITY, J.D.; PAHNKE, M.D.; REESE, E.C.; COYLE, E.F. Maximal mechanical power during a taper in elite swimmers.

Medicine and Science in Sports and Exercise, Madison, v.38, n.9, p.1643-9, 2006.

WILLIAMS, S.J.; KENDALL, L.R. A profile of sports science research (1983-2003). Journal of Science and Medicine in Sport, Belconnen, v.10, n.4, p.193-200, 2007.

YANAI, T. Stroke frequency in front crawl: its mechanical link to the fluid forces required in non-propulsive directions. Journal of Biomechanics, New York, v.36, n.1, p.53-62, 2003.

ENDERECOO

Wilson Viana de Castro Melo Escola Superior de Educação Física Universidade de Pernambuco

R. Arnóbio Marques, 310 50100-130 - Recife - PE - BRASIL e-mail: wviana@usp.br
Recebido para publicação: 01/09/2008

1a. Revisão: 21/01/2009

2a. Revisão: 15/03/2010

Aceito: 28/05/2010 